Original Research Paper

\title{
Minimizing Transmission Time under Required System Capacity Constraint in Stochastic-Flow Networks
}

\author{
Moatamad Refaat Hassan \\ Computer Science Branch, Department of Mathematics, \\ Faculty of Science, Aswan University, Aswan, Egypt
}

\author{
Article history \\ Received: 06-12-2017 \\ Revised: 02-04-2018 \\ Accepted: 04-05-2018 \\ Email: m_r_hassan73@yahoo.com
}

\begin{abstract}
In the case of an existing limited capacity of the system called required system capacity, in this study a new method is presented to determine the disjoint paths to send data from the source to the sink. System reliability is evaluated as the probability that a specified amount of data can be transmitted from a source to a sink through two disjoint paths, subject to a time threshold and a required capacity. In addition, we study the effect of using the proposed method on the reliability evaluation in comparison with other previous methods.
\end{abstract}

Keywords: Quickest Path Problem, Disjoint Paths, Stochastic-Flow Network (SFN)

\section{Introduction}

The QPP is used to find a path that has the minimum transmission time to send a specified amount of data from the source to the sink node (Chin and Chen, 1990; Chen and Hung, 1993). All pairs quickest problem has been solved in (Lee and Papadopoulou, 1993). If the quickest paths are required to go through a specified path, then the restricted problem is called the constrained QPP (Chen and Hung, 1994). Taking into account the lead-time and the capacity of each arc (Martins and Santos, 1997), the problem is solved as a bicriteria path problem, (Sedeno-Noda and Gonzalez-Barrera, 2014). In addition, Lin (2009a) proposed a method based on Monte-Carlo simulation to solve the quickest path flow network reliability problem.

The QPP has been extended to the stochastic-flow network by (Lin, 2003), an algorithm proposed to evaluate the system reliability of a SFN with time consideration. While in (Lin, 2009a), the system reliability has been evaluated under time constraint. In this case, the data are transmitted through two disjoint paths. Lin (2009b) presented an algorithm to determine the optimal routing policy with the highest system reliability. Lin (2010) proposed an algorithm to generate all minimal system states fulfilling the demand, time and budget constraints; then the system reliability is evaluated in terms of such system states. The network reliability has been evaluated in the case of sending units of data through a number of MPs simultaneously under both time and budget constraints (Lin, 2011a). Moreover, they evaluated network reliability according to the spare routing. In order to reduce transmission time, in (Lin, 2011b), a problem of transmitting data through multiple disjoint minimal paths simultaneously was presented. In (Yeh, 2015), an algorithm based on depth-first-search to solve the quickest path reliability problem was proposed. In addition, in (El Khadiri and Yeh, 2016), a method based on Monte-Carlo simulation to solve the quickest path flow network reliability problem was proposed. The required system capacity (Hassan, 2012) has been extended in (Aggarwal et al., 1982) to solve the QPP in a stochastic flow network. Furthermore, the system reliability has been evaluated under time threshold and system capacity constraint.

Previous studies have evaluated the system reliability of an SFN network according to a given demand under the time constraints (Sedeno-Noda and Gonzalez-Barrera, 2014; Lin, 2003; 2009a; 2009b; 2010; 2011a; 2011b; Yeh, 2015; El Khadiri and Yeh, 2016; Yeh et al., 2013; Jane and Laih, 2017). However, these studies have not considered the case of the required system capacity or the system with limited capacity. Therefore, the main purpose of this paper is to study the QPP in an SFN in the case of sending data simultaneously through two DMPs under required system capacity.

The required system capacity has been used to determine the available disjoint paths to send a specified amount of data from the source node to the sink node. Hereby, the set of solutions can be obtained and the system reliability can then be computed in terms of these solutions. The algorithm presented by Lin (2003) can be modified to exploit the idea of using the required system capacity constraint to 
determine the available disjoint paths. The same technique can be used to obtain the set of solutions.

The paper is organized as follows. In section II, the formulation of the problem is presented. System reliability evaluation is given in section III. In section IV, the proposed algorithm to generate all solutions, called Algorithm I is presented. In section V, Illustrative examples are presented to show the efficiency of the proposed algorithm. The computational time of the proposed algorithm is presented in section VI. An important Lemma that can be used to speed up Algorithm I is given in section VII. In section VIII, both the discussion and comparison are presented. In section IX, we provide the conclusion.

\section{Problem formulation}

The transmission time $T P_{j}$ of a path $P_{j}$ is given by:

$$
T P_{j}=\sum_{k=1}^{n}\left\{l_{k} \mid a_{k} \in P_{j}\right\}+\lceil d / v\rceil
$$

where, $v$ is the smallest integer such that $T P_{j} \leq T, T$ is the time threshold.

The maximum capacity $C P_{j}$ of a path $P_{j}$ is given by:

$$
C P_{j}=\operatorname{Min}\left\{M^{k} \mid a_{k} \in P_{j}\right\}
$$

The path $P_{j}$ is said to be the available path under the required system capacity if its maximum capacity is greater than or equal to the required system capacity, i.e., when the condition $C P_{j} \geq C s$ is satisfied. Then the set of available paths $\rho$ is given by:

$\rho=\left\{P_{j} \mid C P_{j} \geq C s, j=1,2, \ldots, n p\right\}$

If no path satisfies the condition $C P_{j} \geq C s$, then $\rho=\varnothing$.

From the set $\rho$, we can construct the set of disjoint paths $\varphi$, where:

$\varphi=\left\{\left(P_{i}, P_{j}\right) \in \rho \mid i \neq j\right.$ and $\left.P_{i} \cap P_{j}=\varnothing\right\}$

The transmission time of sending $d$ units of data simultaneously through two disjoint paths $P_{i}$ and $P_{j}$ under capacity vector $X$ is $\psi\left(d_{i}, X, P_{i}\right)$ and $\psi\left(d_{j}, X, P_{j}\right)$ respectively, where $d_{i}+d_{j}=d$ such that $C P_{i} \geq C s$ and $C P_{j}$ $\geq C s$. Then, $\psi\left(d_{i}, X, P_{i}\right)=\sum_{k=1}^{n} l_{k} \mid a_{k} \in P_{i}+\left\lceil\frac{d_{i}}{C s}\right\rceil$ and $\psi\left(d_{j}\right.$, $\left.X, P_{j}\right)=\sum_{k=1}^{n} l_{k} \mid a_{k} \in P_{j}+\left\lceil\frac{d_{j}}{C s}\right\rceil$. Let $\Gamma$ be the set of candidate solutions $X$ under the above conditions and let $\Gamma_{\min }=\{X \mid X$ isaminimaline $\Gamma\}$. Then, $X \in \Gamma_{\min }$ is called a $(d, T, C s)$-DMPs throughout this paper. For simplicity purposes, let $\mathrm{T}_{\mathrm{i}}$ and $\mathrm{T}_{\mathrm{j}}$ be denoted to $\psi\left(d_{i}, X, P_{i}\right)$ and $\psi\left(d_{j}\right.$, $X, P_{j}$ ) respectively.

\section{Reliability Evaluation}

If $X^{1}, X^{2}, \ldots, X^{b}$ are all $(d, T, C s)$-DMPs obtained, then the system reliability $R_{d, T, C s}$ is given by:

$R_{d, T, C s}=\operatorname{Pr}\left\{\bigcup_{i=1}^{b}\left\{Y \mid Y \geq X^{i}\right\}\right\}$

The above formula can be calculated by the inclusion-exclusion rule (Janan, 1985; Locks, 1980) state space decomposition (Alexopoulos, 1995) and Recursive Sum of Disjoint Products (RSDP) (Zuo et al., 2007), used in this study.

\section{Proposed Algorithm to Generate all $(d, T$, Cs)-DMPs}

The following algorithm uses required value of the Cs to determine the available MPs and then generate all $(d, T, C s)$-DMPs.

Input: A stochastic-flow network and minimal paths, demand level, time limitation and system capacity constraint.

Output: All the $(d, T, C s)$ - DMPs.

Algorithm I: Generate all $(d, T, C s)$-DMPs.

1. Input the paths $P_{i}, i=1,2,3, \ldots, \omega$, demand value and the Cs value.

2. Calculate the minimum capacity for each path $m c p_{i}, C P_{i}=\operatorname{Min}\left\{M_{k} \mid a_{k} \in p_{i}\right\}$.

3. Determine all paths that satisfy the condition $C P_{i} \geq C s$.

4. Construct the set of available disjoint paths $\varphi=$ $\left\{\left(P_{i}, P_{j}\right) \mid i \neq j\right.$ and $\left.P_{i} \cap P_{j}=\varnothing\right\}$ from the paths generated in step 3 .

5. For each disjoint pair $P_{i}$ and $P_{j}$ in $\varphi$ do the following: 5.1. Find the largest demands $\bar{d}_{i}$ and $\bar{d}_{j}$ that can be assigned to $P_{i}$ and $P_{j}$ respectively. Such that:

$$
\sum_{k=1}^{n}\left\{l_{k} \mid a_{k} \in p_{i}\right\}+\left\lceil\bar{d}_{i} / C_{s}\right\rceil \leq T
$$

and:

$$
\sum_{k=1}^{n}\left\{l_{k} \mid a_{k} \in p_{j}\right\}+\left\lceil\bar{d}_{j} / C_{s}\right\rceil \leq T
$$

5.2. Generate the set of solutions:

$$
\beta=\left\{\left(d_{i}, d_{j}\right) \mid d_{i} \leq \bar{d}_{i}, d_{j} \leq \bar{d}_{j} \text { and } d_{i}+d_{j}=d\right\}
$$


5.3. For all solutions in $\beta$ calculate the transmission time for each path:

$$
T_{i}=\sum_{k=1}^{n}\left\{l_{k} \mid a_{k} \in p_{i}\right\}+\left\lceil d_{i} / C_{s}\right\rceil
$$

and:

$$
T_{j}=\sum_{k=1}^{n}\left\{l_{k} \mid a_{k} \in p_{j}\right\}+\left\lceil d_{j} / C_{s}\right\rceil
$$

5.4. If $T_{i} \leq T$ and $T_{j} \leq T$, then generate the capacity vector $X^{q}=\left(x_{1}, x_{2}, \ldots, x_{k}, \ldots, x_{n}\right)$ as follows:

$$
x_{k}=\left\{\begin{array}{cc}
C s & \text { if } a_{k} \in P_{i} \cup P_{j} \\
0 & \text { Otherwise }
\end{array}\right.
$$

5.5. Repeat steps 5.3 and 5.4 to generate all solutions.

5.6. Remove the solutions that are not lower.

6. Evaluate the system reliability for the generated lower capacity vectors.

7. End

\section{Illustrative Examples}

\section{A. Five Nodes Network Example}

The network of Fig. 1 has five nodes and eight arcs, (Lin, 2009a). The information of each arc is given in Table 1. The Minimal paths and their maximum capacity are given in Table 2. The available disjoint minimal paths corresponding to each value for $\mathrm{Cs}$, the available values for $\left(d_{1}, d_{2}\right)$, the corresponding $(d, T, C s)$-DMP solutions and $R_{d, T, C s}$ are given in Table 3 . The best solutions found are given in Table 4. The demand $d$ equals to 8 and the time limit is 9 for this example.

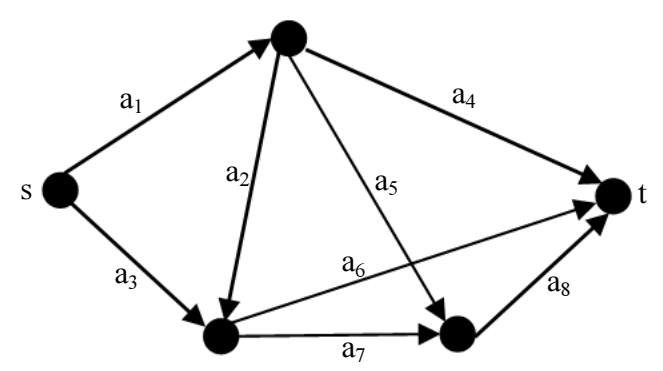

\begin{tabular}{|c|c|c|c|c|c|c|c|}
\hline Arc & Capacity & Probability & Lead time & Arc & Capacity & Probability & Lead time \\
\hline \multirow[t]{4}{*}{$a_{1}$} & 0 & 0.05 & 2 & $a_{5}$ & 0 & 0.10 & 1 \\
\hline & 1 & 0.05 & & & 1 & 0.10 & \\
\hline & 2 & 0.10 & & & 2 & 0.80 & \\
\hline & 3 & 0.80 & & & & & \\
\hline \multirow[t]{5}{*}{$\mathrm{a}_{2}$} & 0 & 0.05 & 1 & $a_{6}$ & 0 & 0.05 & 2 \\
\hline & 1 & 0.05 & & & 1 & 0.05 & \\
\hline & 2 & 0.10 & & & 2 & 0.10 & \\
\hline & 3 & 0.80 & & & 3 & 0.20 & \\
\hline & & & & & 4 & 0.60 & \\
\hline \multirow[t]{6}{*}{$a_{3}$} & 0 & 0.05 & 3 & $a_{7}$ & 0 & 0.05 & 2 \\
\hline & 1 & 0.05 & & & 1 & 0.10 & \\
\hline & 2 & 0.10 & & & 2 & 0.10 & \\
\hline & 3 & 0.80 & & & 3 & 0.10 & \\
\hline & & & & & 4 & 0.10 & \\
\hline & & & & & 5 & 0.55 & \\
\hline \multirow[t]{5}{*}{$\mathrm{a}_{4}$} & 0 & 0.10 & 3 & $\mathrm{a}_{8}$ & 0 & 0.05 & 1 \\
\hline & 1 & 0.90 & & & 1 & 0.05 & \\
\hline & & & & & 2 & 0.10 & \\
\hline & & & & & 3 & 0.10 & \\
\hline & & & & & 4 & 0.70 & \\
\hline
\end{tabular}

Fig. 1: Computer network

Table 1: Arcs information

Table 2: The paths and their corresponding maximum capacity

\begin{tabular}{lll}
\hline No. & $\mathrm{P}_{\mathrm{i}}$ & $\mathrm{CP}$ \\
\hline 1 & $\left\{\mathrm{a}_{1}, \mathrm{a}_{4}\right\}$ & 1 \\
2 & $\left\{\mathrm{a}_{1}, \mathrm{a}_{5}, \mathrm{a}_{8}\right\}$ & 2 \\
3 & $\left\{\mathrm{a}_{1}, \mathrm{a}_{2}, \mathrm{a}_{6}\right\}$ & 3 \\
4 & $\left\{\mathrm{a}_{1}, \mathrm{a}_{2}, \mathrm{a}_{7}, \mathrm{a}_{8}\right\}$ & 3 \\
5 & $\left\{\mathrm{a}_{3}, \mathrm{a}_{6}\right\}$ & 3 \\
6 & $\left\{\mathrm{a}_{3}, \mathrm{a}_{7}, \mathrm{a}_{8}\right\}$ & 3 \\
\hline
\end{tabular}


Table 3: The results for different values of Cs

\begin{tabular}{|c|c|c|c|c|c|c|c|c|c|c|c|}
\hline \multirow{2}{*}{$\begin{array}{ll}\text { Cs } \\
1\end{array}$} & \multirow{2}{*}{$\begin{array}{l}\text { Disjoint pair } \\
\mathrm{P}_{1}=\left\{\mathrm{a}_{1}, \mathrm{a}_{4}\right\} \text { and } \mathrm{P}_{5}=\left\{\mathrm{a}_{3}, \mathrm{a}_{6}\right\}\end{array}$} & \multirow{2}{*}{$\frac{\left(d_{1}, d_{2}\right)}{(4,4)}$} & \multicolumn{8}{|c|}{ The capacity vector $\mathrm{X}$} & \multirow{2}{*}{$\begin{array}{l}\mathrm{R}_{\mathrm{d}, \mathrm{T}, \mathrm{Cs}} \\
0.771638\end{array}$} \\
\hline & & & 1 & 0 & 1 & 1 & 0 & 1 & 0 & 0 & \\
\hline & $P_{1}=\left\{a_{1}, a_{4}\right\}$ and $P_{6}=\left\{a_{3}, a_{7}, a_{8}\right\}$ & $*$ & - & & & & & & & & - \\
\hline & $\mathrm{P}_{2}=\left\{\mathrm{a}_{1}, \mathrm{a}_{5}, \mathrm{a}_{8}\right\}$ and $\mathrm{P}_{5}=\left\{\mathrm{a}_{3}, \mathrm{a}_{6}\right\}$ & $(4,4)$ & 1 & 0 & 1 & 0 & 1 & 1 & 0 & 1 & 0.733056 \\
\hline \multirow[t]{23}{*}{2} & $\mathrm{P}_{2}=\left\{\mathrm{a}_{1}, \mathrm{a}_{5}, \mathrm{a}_{8}\right\}$ and $\mathrm{P}_{5}=\left\{\mathrm{a}_{3}, \mathrm{a}_{6}\right\}$ & $(8,0)$ & 2 & 0 & 0 & 0 & 2 & 0 & 0 & 2 & 0.93312 \\
\hline & & $(7,1)$ & 2 & 0 & 2 & 0 & 2 & 2 & 0 & 2 & \\
\hline & & $(6,2)$ & 2 & 0 & 2 & 0 & 2 & 2 & 0 & 2 & \\
\hline & & $(5,3)$ & 2 & 0 & 2 & 0 & 2 & 2 & 0 & 2 & \\
\hline & & $(4,4)$ & 2 & 0 & 2 & 0 & 2 & 2 & 0 & 2 & \\
\hline & & $(3,5)$ & 2 & 0 & 2 & 0 & 2 & 2 & 0 & 2 & \\
\hline & & $(2,6)$ & 2 & 0 & 2 & 0 & 2 & 2 & 0 & 2 & \\
\hline & & $(1,7)$ & 2 & 0 & 2 & 0 & 2 & 2 & 0 & 2 & \\
\hline & & $(0,8)$ & 0 & 0 & 2 & 0 & 0 & 2 & 0 & 0 & \\
\hline & $P_{3}=\left\{a_{1}, a_{2}, a_{6}\right\}$ and $P_{6}=\left\{a_{3}, a_{7}, a_{8}\right\}$ & $(8,0)$ & 2 & 2 & 0 & 0 & 0 & 2 & 0 & 0 & 0.729 \\
\hline & & $(7,1)$ & 2 & 2 & 2 & 0 & 0 & 2 & 2 & 2 & \\
\hline & & $(6,2)$ & 2 & 2 & 2 & 0 & 0 & 2 & 2 & 2 & \\
\hline & & $(5,3)$ & 2 & 2 & 2 & 0 & 0 & 2 & 2 & 2 & \\
\hline & & $(4,4)$ & 2 & 2 & 2 & 0 & 0 & 2 & 2 & 2 & \\
\hline & & $(3,5)$ & 2 & 2 & 2 & 0 & 0 & 2 & 2 & 2 & \\
\hline & & $(2,6)$ & 2 & 2 & 2 & 0 & 0 & 2 & 2 & 2 & \\
\hline & $P_{4}=\left\{a_{1}, a_{2}, a_{7}, a_{8}\right\}$ and $P_{5}=\left\{a_{3}, a_{6}\right\}$ & $(6,2)$ & 2 & 2 & 2 & 0 & 0 & 2 & 2 & 2 & 0.81 \\
\hline & & $(5,3)$ & 2 & 2 & 2 & 0 & 0 & 2 & 2 & 2 & \\
\hline & & $(4,4)$ & 2 & 2 & 2 & 0 & 0 & 2 & 2 & 2 & \\
\hline & & $(3,5)$ & 2 & 2 & 2 & 0 & 0 & 2 & 2 & 2 & \\
\hline & & $(2,6)$ & 2 & 2 & 2 & 0 & 0 & 2 & 2 & 2 & \\
\hline & $(1,7)$ & 2 & 2 & 2 & 0 & 0 & 2 & 2 & 2 & & \\
\hline & & $(0,8)$ & 0 & 0 & 2 & 0 & 0 & 2 & 0 & 0 & \\
\hline \multirow[t]{18}{*}{3} & $\mathrm{P}_{3}=\left\{\mathrm{a}_{1}, \mathrm{a}_{2}, \mathrm{a}_{6}\right\}$ and & $(8,0)$ & 3 & 3 & 0 & 0 & 0 & 3 & 0 & 0 & 0.74624 \\
\hline & $\mathrm{P}_{6}=\left\{\mathrm{a}_{3}, \mathrm{a}_{7}, \mathrm{a}_{8}\right\}$ & $(7,1)$ & 3 & 3 & 3 & 0 & 0 & 3 & 3 & 3 & \\
\hline & & $(6,2)$ & 3 & 3 & 3 & 0 & 0 & 3 & 3 & 3 & \\
\hline & & $(5,3)$ & 3 & 3 & 3 & 0 & 0 & 3 & 3 & 3 & \\
\hline & & $(4,4)$ & 3 & 3 & 3 & 0 & 0 & 3 & 3 & 3 & \\
\hline & & $(3,5)$ & 3 & 3 & 3 & 0 & 0 & 3 & 3 & 3 & \\
\hline & & $(2,6)$ & 3 & 3 & 3 & 0 & 0 & 3 & 3 & 3 & \\
\hline & & $(1,7)$ & 3 & 3 & 3 & 0 & 0 & 3 & 3 & 3 & \\
\hline & & $(0,8)$ & 0 & 0 & 3 & 0 & 0 & 0 & 3 & 3 & \\
\hline & $\mathrm{P}_{4}=\left\{\mathrm{a}_{1}, \mathrm{a}_{2}, \mathrm{a}_{7}, \mathrm{a}_{8}\right\}$ and & $(8,0)$ & 3 & 3 & 0 & 0 & 0 & 0 & 3 & 3 & 0.77824 \\
\hline & $\mathrm{P}_{5}=\left\{\mathrm{a}_{3}, \mathrm{a}_{6}\right\}$ & $(7,1)$ & 3 & 3 & 3 & 0 & 0 & 3 & 3 & 3 & \\
\hline & & $(6,2)$ & 3 & 3 & 3 & 0 & 0 & 3 & 3 & 3 & \\
\hline & & $(5,3)$ & 3 & 3 & 3 & 0 & 0 & 3 & 3 & 3 & \\
\hline & & $(4,4)$ & 3 & 3 & 3 & 0 & 0 & 3 & 3 & 3 & \\
\hline & & $(3,5)$ & 3 & 3 & 3 & 0 & 0 & 3 & 3 & 3 & \\
\hline & & $(2,6)$ & 3 & 3 & 3 & 0 & 0 & 3 & 3 & 3 & \\
\hline & & $(1,7)$ & 3 & 3 & 3 & 0 & 0 & 3 & 3 & 3 & \\
\hline & & $(0,8)$ & 0 & 0 & 3 & 0 & 0 & 3 & 0 & 0 & \\
\hline
\end{tabular}

*Means that $(\mathrm{d} 1, \mathrm{~d} 2)$ does not satisfy one or more conditions mentioned in the algorithm. Also for the disjoint pairs \{P3,P6\} and $\{\mathrm{P} 4, \mathrm{P} 5\}$

Table 4: The best disjoint paths for each Cs value

\begin{tabular}{lll}
\hline Cs & The best disjoint pair & $\mathrm{R}_{\mathrm{d}, \mathrm{T}, \mathrm{Cs}}$ \\
\hline 1 & $\mathrm{P}_{1}=\left\{\mathrm{a}_{1}, \mathrm{a}_{4}\right\}$ and $\mathrm{P}_{5}=\left\{\mathrm{a}_{3}, \mathrm{a}_{6}\right\}$ & 0.771638 \\
2 & $\mathrm{P}_{2}=\left\{\mathrm{a}_{1}, \mathrm{a}_{5}, \mathrm{a}_{8}\right\}$ and $\mathrm{P}_{5}=\left\{\mathrm{a}_{3}, \mathrm{a}_{6}\right\}$ & 0.933120 \\
3 & $\mathrm{P}_{4}=\left\{\mathrm{a}_{1}, \mathrm{a}_{2}, \mathrm{a}_{7}, \mathrm{a}_{8}\right\}$ and $\mathrm{P}_{5}=\left\{\mathrm{a}_{3}, \mathrm{a}_{6}\right\}$ & 0.778240 \\
\hline
\end{tabular}

\section{B. Fourteen Nodes Network Example}

The network given in Fig. 2 is taken from (Lin, 2011a). The arcs' information is given in Table 5. The network contains eleven paths shown in Table 6. Also, the maximal capacity for each path has been determined. The available paths for different values of Cs are shown in Table 7. The best disjoint pair of paths for each Cs shown in Table 8 . 
Moatamad Refaat Hassan / American Journal of Applied Sciences 2018, 15 (5): 251.260 DOI: 10.3844/ajassp.2018.251.260

Table 5: Arcs information

\begin{tabular}{|c|c|c|c|c|c|c|c|}
\hline Arc & Capacity & Probability & Lead time & Arc & Capacity & Probability & Lead time \\
\hline \multirow[t]{5}{*}{$a_{1}$} & 0 & 0.06 & 2 & $a_{12}$ & 0 & 0.04 & 2 \\
\hline & 10 & 0.03 & & & 10 & 0.05 & \\
\hline & 30 & 0.05 & & & 20 & 0.04 & \\
\hline & 50 & 0.86 & & & 40 & 0.04 & \\
\hline & & & & & 60 & 0.83 & \\
\hline \multirow[t]{5}{*}{$a_{2}$} & 0 & 0.05 & 2 & $a_{13}$ & 0 & 0.04 & 1 \\
\hline & 10 & 0.02 & & & 10 & 0.02 & \\
\hline & 30 & 0.03 & & & 20 & 0.02 & \\
\hline & 50 & 0.9 & & & 40 & 0.01 & \\
\hline & & & & & 60 & 0.82 & \\
\hline \multirow[t]{4}{*}{$a_{3}$} & 0 & 0.04 & 3 & $a_{14}$ & 0 & 0.05 & 2 \\
\hline & 10 & 0.04 & & & 20 & 0.95 & \\
\hline & 20 & 0.04 & & & & & \\
\hline & 40 & 0.88 & & & & & \\
\hline \multirow[t]{5}{*}{$\mathrm{a} 4$} & 0 & 0.05 & 3 & $a_{15}$ & 0 & 0.05 & 3 \\
\hline & 10 & 0.05 & & & 10 & 0.05 & \\
\hline & 30 & 0.05 & & & 30 & 0.05 & \\
\hline & 50 & 0.85 & & & 50 & 0.05 & \\
\hline & & & & & 70 & 0.8 & \\
\hline \multirow[t]{5}{*}{ a5 } & 0 & 0.05 & 4 & $a_{16}$ & 0 & 0.04 & 2 \\
\hline & 10 & 0.05 & & & 10 & 0.04 & \\
\hline & 30 & 0.05 & & & 30 & 0.04 & \\
\hline & 50 & 0.85 & & & 40 & 0.06 & \\
\hline & & & & & 60 & 0.82 & \\
\hline \multirow[t]{4}{*}{ a6 } & 0 & 0.04 & 3 & $a_{17}$ & 0 & 0.05 & 3 \\
\hline & 10 & 0.04 & & & 10 & 0.04 & \\
\hline & 20 & 0.04 & & & 30 & 0.03 & \\
\hline & 40 & 0.88 & & & 50 & 0.88 & \\
\hline \multirow[t]{4}{*}{$a_{7}$} & 0 & 0.04 & 2 & $a_{18}$ & 0 & 0.05 & 3 \\
\hline & 20 & 0.96 & & & 10 & 0.05 & \\
\hline & & & & & 30 & 0.05 & \\
\hline & & & & & 40 & 0.85 & \\
\hline \multirow[t]{4}{*}{$\mathrm{a}_{8}$} & 0 & 0.05 & 3 & $a_{19}$ & 0 & 0.04 & 3 \\
\hline & 10 & 0.05 & & & 10 & 0.03 & \\
\hline & 30 & 0.05 & & & 30 & 0.03 & \\
\hline & 50 & 0.85 & & & 50 & 0.9 & \\
\hline \multirow[t]{4}{*}{$\mathrm{a}_{9}$} & 0 & 0.05 & 4 & $a_{20}$ & 0 & 0.05 & 3 \\
\hline & 10 & 0.05 & & & 10 & 0.05 & \\
\hline & 20 & 0.07 & & & 20 & 0.1 & \\
\hline & 40 & 0.83 & & & 40 & 0.8 & \\
\hline \multirow[t]{4}{*}{$\mathrm{a}_{10}$} & 0 & 0.05 & 2 & $a_{21}$ & 0 & 0.07 & 2 \\
\hline & 10 & 0.05 & & & 20 & 0.93 & \\
\hline & 20 & 0.05 & & & & & \\
\hline & 40 & 0.85 & & & & & \\
\hline \multirow[t]{4}{*}{$a_{11}$} & 0 & 0.05 & 3 & $a_{22}$ & 0 & 0.04 & 4 \\
\hline & 10 & 0.05 & & & 10 & 0.96 & \\
\hline & 30 & 0.02 & & & & & \\
\hline & 50 & 0.88 & & & & & \\
\hline
\end{tabular}

Table 6: The paths and their maximum capacity

\begin{tabular}{|c|c|c|}
\hline No. & $P_{j}$ & $\mathrm{CP}_{\mathrm{j}}$ \\
\hline 1 & $\left\{a_{1}, a_{2}, a_{3}\right\}$ & 40 \\
\hline 2 & $\left\{a_{1}, a_{6}, a_{7}\right\}$ & 20 \\
\hline 3 & $\left\{a_{4}, a_{5}, a_{6}\right\}$ & 40 \\
\hline 4 & $\left\{a_{8}, a_{9}, a_{10}\right\}$ & 40 \\
\hline 5 & $\left\{a_{10}, a_{11}, a_{14}\right\}$ & 20 \\
\hline 6 & $\left\{a_{11}, a_{12}, a_{13}\right\}$ & 50 \\
\hline 7 & $\left\{\mathrm{a}_{15}, \mathrm{a}_{22}\right\}$ & 10 \\
\hline 8 & $\left\{a_{15}, a_{16}, a_{17}\right\}$ & 50 \\
\hline 9 & $\left\{\mathrm{a}_{18}, \mathrm{a}_{21}, \mathrm{a}_{22}\right\}$ & 10 \\
\hline 10 & $\left\{\mathrm{a}_{16}, \mathrm{a}_{17}, \mathrm{a}_{18}, \mathrm{a}_{21}\right\}$ & 20 \\
\hline 11 & $\left\{\mathrm{a}_{18}, \mathrm{a}_{19}, \mathrm{a}_{20}\right\}$ & 40 \\
\hline
\end{tabular}


Table 7: The available paths for different values of Cs

\begin{tabular}{|c|c|c|c|c|c|}
\hline Cs & $P_{i}$ & Cs & $P_{j}$ & Cs & $\mathrm{P}_{\mathrm{j}}$ \\
\hline 更 & $\begin{array}{l}\left\{a_{11}, a_{12}, a_{13}\right\} \\
\left\{a_{15}, a_{16}, a_{17}\right\}\end{array}$ & 20 & $\begin{array}{l}\left\{\mathrm{a}_{11}, \mathrm{a}_{12}, \mathrm{a}_{13}\right\} \\
\left\{\mathrm{a}_{15}, \mathrm{a}_{16}, \mathrm{a}_{17}\right\} \\
\left\{\mathrm{a}_{1}, \mathrm{a}_{2}, \mathrm{a}_{3}\right\} \\
\left\{\mathrm{a}_{4}, \mathrm{a}_{5}, \mathrm{a}_{6}\right\} \\
\left\{\mathrm{a}_{8}, \mathrm{a}_{9}, \mathrm{a}_{10}\right\} \\
\left\{\mathrm{a}_{18}, \mathrm{a}_{19}, \mathrm{a}_{20}\right\} \\
\left\{\mathrm{a}_{1}, \mathrm{a}_{6}, \mathrm{a}_{7}\right\} \\
\left\{\mathrm{a}_{10}, \mathrm{a}_{11}, \mathrm{a}_{14}\right\} \\
\left\{\mathrm{a}_{16}, \mathrm{a}_{17}, \mathrm{a}_{18}, \mathrm{a}_{21}\right\}\end{array}$ & 10 & $\begin{array}{l}\left\{a_{1}, a_{2}, a_{3}\right\} \\
\left\{a_{1}, a_{6}, a_{7}\right\} \\
\left\{a_{4}, a_{5}, a_{6}\right\} \\
\left\{a_{8}, a_{9}, a_{10}\right\} \\
\left\{a_{10}, a_{11}, a_{14}\right\} \\
\left\{a_{11}, a_{12}, a_{13}\right\} \\
\left\{a_{15}, a_{22}\right\} \\
\left\{a_{15}, a_{16}, a_{17}\right\} \\
\left\{a_{18}, a_{21}, a_{22}\right\} \\
\left\{a_{16}, a_{17}, a_{18}, a_{21}\right\}\end{array}$ \\
\hline 40 & $\begin{array}{l}\left\{a_{11}, a_{12}, a_{13}\right\} \\
\left\{a_{15}, a_{16}, a_{17}\right\} \\
\left\{a_{1}, a_{2}, a_{3}\right\} \\
\left\{a_{4}, a_{5}, a_{6}\right\} \\
\left\{a_{8}, a_{9}, a_{10}\right\} \\
\left\{a_{18}, a_{19}, a_{20}\right\}\end{array}$ & & & & $\left\{\mathrm{a}_{18}, \mathrm{a}_{19}, \mathrm{a}_{20}\right\}$ \\
\hline
\end{tabular}

Table 8: The best disjoint paths for each Cs

\begin{tabular}{lll}
\hline Cs & The best disjoint pair & $\mathrm{R}_{\mathrm{d}, \mathrm{T}, \mathrm{Cs}}$ \\
\hline 10 & $\mathrm{P}_{1}=\left\{\mathrm{a}_{1}, \mathrm{a}_{2}, \mathrm{a}_{3}\right\}$ and $\mathrm{P}_{6}=\left\{\mathrm{a}_{11}, \mathrm{a}_{12}, \mathrm{a}_{13}\right\}$ & 0.91 \\
20 & $\mathrm{P}_{1}=\left\{\mathrm{a}_{1}, \mathrm{a}_{2}, \mathrm{a}_{3}\right\}$ and $\mathrm{P}_{6}=\left\{\mathrm{a}_{11}, \mathrm{a}_{12}, \mathrm{a}_{13}\right\}$ & 0.91 \\
40 & $\mathrm{P}_{1}=\left\{\mathrm{a}_{1}, \mathrm{a}_{2}, \mathrm{a}_{3}\right\}$ and $\mathrm{P}_{6}=\left\{\mathrm{a}_{11}, \mathrm{a}_{12}, \mathrm{a}_{13}\right\}$ & 0.822451 \\
50 & $\mathrm{P}_{6}=\left\{\mathrm{a}_{11}, \mathrm{a}_{12}, \mathrm{a}_{13}\right\}$ and $\mathrm{P}_{8}=\left\{\mathrm{a}_{15}, \mathrm{a}_{16}, \mathrm{a}_{17}\right\}$ & 0.789727 \\
\hline
\end{tabular}

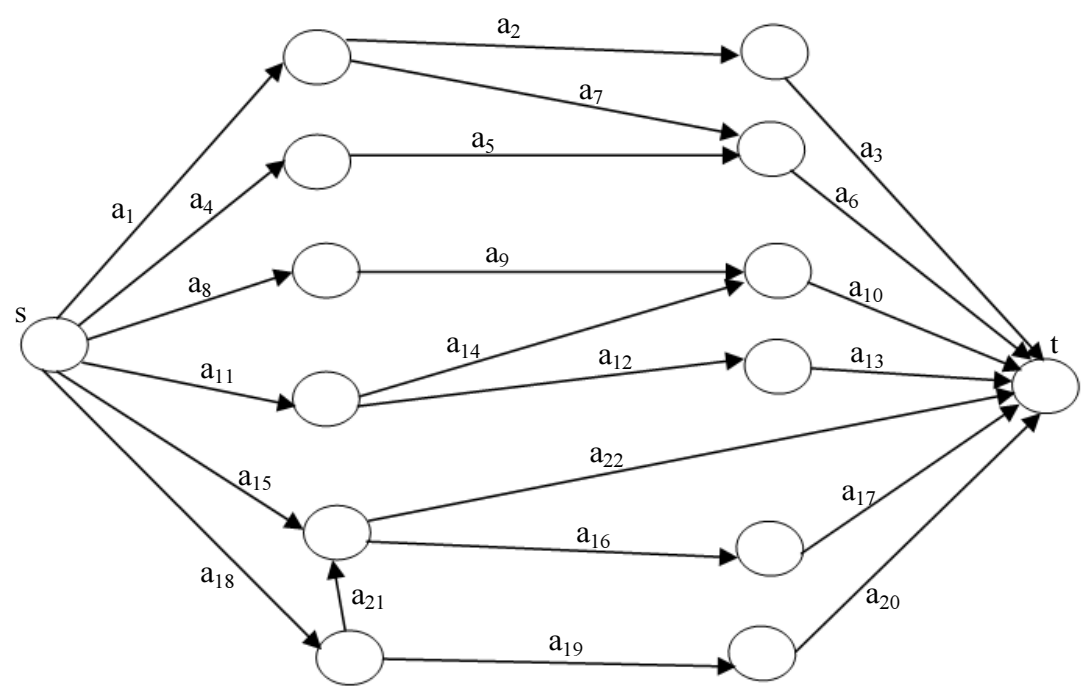

Fig. 2: Example of a Network of fourteen nodes

\section{Time Analysis}

The algorithm Computes the maximum capacity of each path in $O(\omega n)$ time and $O(\omega)$ to determine the set of disjoint paths. The time needed to determine $\bar{d}_{1}$ and $\bar{d}_{2}$ is $\mathrm{O}(\propto n)$, where $\propto$ represents the number of disjoint paths. To test transmission time, it takes $\mathrm{O}(\propto n)$. To generate all $(d, T, \mathrm{Cs})$-DMPs, it takes $O(\propto d n)$ time. Then, the total time is $O(\delta n)$ taken by the proposed algorithm to find $\Gamma_{\min }$, where $\delta=\omega+(2+d) \alpha$.

\section{An Important Lemma}

The time needed to compare each candidate solution $X$ with all other solutions to construct $\Gamma_{\min }$ is $O(d n)$, in the worst case scenario. This time can be decreased by using the following Lemma.

\section{Lemma 1}

If $X$ is $(d, T)$-DP candidate under $\mathrm{Cs}$ and $X$ corresponds to the solution $\left(d_{1}, 0\right)$ or $\left(0, d_{2}\right)$, then $X$ is a lower vector. 
Table 9: The lower vectors in example A according to Lemma 1

\begin{tabular}{|c|c|c|c|c|c|c|c|c|c|c|c|}
\hline \multirow{2}{*}{$\frac{\text { Cs }}{2}$} & \multirow{2}{*}{$\begin{array}{l}\text { The disjoint pair } \\
\mathrm{P}_{2}=\left\{\mathrm{a}_{1}, \mathrm{a}_{5}, \mathrm{a}_{8}\right\} \text { and } \mathrm{P}_{5}=\left\{\mathrm{a}_{3}, \mathrm{a}_{6}\right\}\end{array}$} & \multirow{2}{*}{$\frac{\left(d_{1}, d_{2}\right)}{(8,0)}$} & \multicolumn{8}{|c|}{ The lower vectors } & \multirow{2}{*}{$\begin{array}{l}\mathrm{R}_{\mathrm{d}, \mathrm{T}, \mathrm{Cs}} \\
0.93312\end{array}$} \\
\hline & & & 2 & 0 & 0 & 0 & 2 & 0 & 0 & 2 & \\
\hline \multirow{7}{*}{3} & & $(0,8)$ & 0 & 0 & 2 & 0 & 0 & 2 & 0 & 0 & \\
\hline & $P_{3}=\left\{a_{1}, a_{2}, a_{6}\right\}$ and $P_{6}=\left\{a_{3}, a_{7}, a_{8}\right\}$ & $(8,0)$ & 2 & 2 & 0 & 0 & 0 & 2 & 0 & 0 & 0.729 \\
\hline & $\mathrm{P}_{4}=\left\{\mathrm{a}_{1}, \mathrm{a}_{2}, \mathrm{a}_{7}, \mathrm{a}_{8}\right\}$ and $\mathrm{P}_{5}=\left\{\mathrm{a}_{3}, \mathrm{a}_{6}\right\}$ & $(8,0)$ & 0 & 0 & 2 & 0 & 0 & 2 & 0 & 0 & 0.81 \\
\hline & $\mathrm{P}_{3}=\left\{\mathrm{a}_{1}, \mathrm{a}_{2}, \mathrm{a}_{6}\right\}$ and & $(8,0)$ & 3 & 3 & 0 & 0 & 0 & 3 & 0 & 0 & 0.74624 \\
\hline & $\mathrm{P}_{6}=\left\{\mathrm{a}_{3}, \mathrm{a}_{7}, \mathrm{a}_{8}\right\}$ & $(0,8)$ & 0 & 0 & 3 & 0 & 0 & 0 & 3 & 3 & \\
\hline & $\mathrm{P}_{4}=\left\{\mathrm{a}_{1}, \mathrm{a}_{2}, \mathrm{a}_{7}, \mathrm{a}_{8}\right\}$ and & $(8,0)$ & 3 & 3 & 0 & 0 & 0 & 0 & 3 & 3 & 0.77824 \\
\hline & $\mathrm{P}_{5}=\left\{\mathrm{a}_{3}, \mathrm{a}_{6}\right\}$ & $(0,8)$ & 0 & 0 & 3 & 0 & 0 & 3 & 0 & 0 & \\
\hline
\end{tabular}

\section{Proof}

Let $P_{1}=\left\{\mathrm{a}_{1}, \mathrm{a}_{4}\right\}$ and $P_{2}=\left\{\mathrm{a}_{3}, \mathrm{a}_{6}\right\}$ and $C s=V$. Let $X^{1}=\left(\begin{array}{llllll}V & 0 & 0 & V & 0 & 0\end{array}\right)$ and $X^{2}=\left(\begin{array}{llllll}0 & 0 & V & 0 & 0 & V\end{array}\right)$ are $(d, T)$ DMP candidate to $(d 1,0)$ and $(0, d 2)$ respectively. Then all other (d, T)-DMP candidates will be in the form $(V$ $0 V V 0 V$ ). It is easy to verify that $X^{1}$ and $X^{2}$ are lower. Table 9 shows lower vectors based on using this Lemma in Example A.

\section{Discussion and Comparison}

In this section, we investigate how to use the system capacity constraint to determine the candidate disjoint paths and then use Lin's Algorithm presented in (Lin, 2009a) to obtain the solutions, given by Algorithm II. In addition, the results of applying Algorithm II on both Example A and Example Bare shown in Table 10 to 12 . While Table 13 gives the comparison between the results obtained by the Algorithm I and that obtained by Algorithm II to show the difference to the reliability without using Cs value to deduce the solutions.

Algorithm II: Using $C s$ to the available MPs and using Lin's Algorithm to generate all (d, T)DMPs.

1. Input the paths $P_{i}, i=1,2,3, \ldots, \omega$ and the Cs value.

2. Calculate the maximum capacity for each path $C P_{i}$, $C P_{j}=\operatorname{Min}\left\{M_{k} \mid a_{k} \in p_{j}\right\}$.

3. Determine all paths that satisfy the condition $C P_{i}$ $\geq C s$.

4. Construct the set of available disjoint paths $\varphi=$ $\left\{\left(P_{i}, P_{j}\right) \mid i \neq j\right.$ and $\left.P_{i} \cap P_{j}=\varnothing\right\}$ from the paths generated in step 3 .

5. For each disjoint pair $P_{i}$ and $P_{j}$ in $\varphi$, do the following (Lin, 2009a):

5.1. Find the largest demands $\bar{d}_{i}$ and $\bar{d}_{j}$ that can be assigned to $P_{i}$ and $P_{j}$ respectively, such that:

$$
\sum_{k=1}^{n}\left\{l_{k} \mid a_{k} \in p_{i}\right\}+\left\lceil\bar{d}_{i} / C P_{i}\right\rceil \leq T
$$

and:

$$
\sum_{k=1}^{n}\left\{l_{k} \mid a_{k} \in p_{j}\right\}+\left\lceil\bar{d}_{j} / C P_{j}\right\rceil \leq T
$$

5.2. Generate the set of solutions $\beta=\left\{\left(d_{i}, d_{j}\right) \mid d_{i} \leq \bar{d}_{i}, d_{j} \leq \bar{d}_{j}\right.$ and $\left.d_{i}+d_{j}=d\right\}$

5.3. For all solutions in $\beta$, find the smallest integers $v_{\mathrm{i}}$ and $v_{j}$, such that the transmission time for each path is calculated as:

$$
\sum_{k=1}^{n}\left\{l_{k} \mid a_{k} \in p_{i}\right\}+\left\lceil d_{i} / v_{i}\right\rceil \leq T
$$

and:

$$
\sum_{k=1}^{n}\left\{l_{k} \mid a_{k} \in p_{j}\right\}+\left\lceil d_{j} / v_{j}\right\rceil \leq T
$$

5.4. Generate the capacity vector $X^{q}=\left(x_{1}, x_{2}, \ldots\right.$, $\left.x_{k}, \ldots, x_{n}\right)$ as follows:

$$
x_{k}=\left\{\begin{array}{cc}
u_{k} \geq v_{i} & \text { if } a_{k} \in P_{i} \\
u_{k} \geq v_{j} & \text { if } a_{k} \in P_{j} \\
0 & \text { Otherwise }
\end{array}\right.
$$

5.5. Repeat steps 5.3 and 5.4 to generate all solutions.

5.6. Remove the solutions that are not lower.

6. Evaluate the system reliability for the generated lower capacity vectors.

7. End

The results in Table 13 show that the system reliability values are affected by using Cs value to generate solutions. The results obtained by using both Lin's Algorithm and Algorithm II are identical. However, Algorithm II can be used to determine the available disjoint paths and then generate the solutions. 
Table 10: The results of applying algorithm II to example A

\begin{tabular}{llr}
\hline Cs & The disjoint pair & $\mathrm{R}_{\mathrm{d}, \mathrm{T}, \mathrm{Cs}}$ \\
\hline 1 & $\mathrm{P}_{1}=\left\{\mathrm{a}_{1}, \mathrm{a}_{4}\right\}$ and $\mathrm{P}_{5}=\left\{\mathrm{a}_{3}, \mathrm{a}_{6}\right\}$ & 0.889088 \\
& $\mathrm{P}_{1}=\left\{\mathrm{a}_{1}, \mathrm{a}_{4}\right\}$ and $\mathrm{P}_{6}=\left\{\mathrm{a}_{3}, \mathrm{a}_{7}, \mathrm{a}_{8}\right\}$ & 0.658268 \\
& $\mathrm{P}_{2}=\left\{\mathrm{a}_{1}, \mathrm{a}_{5}, \mathrm{a}_{8}\right\}$ and $\mathrm{P}_{5}=\left\{\mathrm{a}_{3}, \mathrm{a}_{6}\right\}$ & 0.948313 \\
& $\mathrm{P}_{3}=\left\{\mathrm{a}_{1}, \mathrm{a}_{2}, \mathrm{a}_{6}\right\}$ and $\mathrm{P}_{6}=\left\{\mathrm{a}_{3}, \mathrm{a}_{7}, \mathrm{a}_{8}\right\}$ & 0.885846 \\
& $\mathrm{P}_{4}=\left\{\mathrm{a}_{1}, \mathrm{a}_{2}, \mathrm{a}_{7}, \mathrm{a}_{8}\right\}$ and $\mathrm{P}_{5}=\left\{\mathrm{a}_{3}, \mathrm{a}_{6}\right\}$ & 0.904758 \\
& $\mathrm{P}_{2}=\left\{\mathrm{a}_{1}, \mathrm{a}_{5}, \mathrm{a}_{8}\right\}$ and $\mathrm{P}_{5}=\left\{\mathrm{a}_{3}, \mathrm{a}_{6}\right\}$ & 0.948313 \\
& $\mathrm{P}_{3}=\left\{\mathrm{a}_{1}, \mathrm{a}_{2}, \mathrm{a}_{6}\right\}$ and $\mathrm{P}_{6}=\left\{\mathrm{a}_{3}, \mathrm{a}_{7}, \mathrm{a}_{8}\right\}$ & 0.885846 \\
3 & $\mathrm{P}_{4}=\left\{\mathrm{a}_{1}, \mathrm{a}_{2}, \mathrm{a}_{7}, \mathrm{a}_{8}\right\}$ and $\mathrm{P}_{5}=\left\{\mathrm{a}_{3}, \mathrm{a}_{6}\right\}$ & 0.904758 \\
& $\mathrm{P}_{3}=\left\{\mathrm{a}_{1}, \mathrm{a}_{2}, \mathrm{a}_{6}\right\}$ and $\mathrm{P}_{6}=\left\{\mathrm{a}_{3}, \mathrm{a}_{7}, \mathrm{a}_{8}\right\}$ & 0.885846 \\
& $\mathrm{P}_{4}=\left\{\mathrm{a}_{1}, \mathrm{a}_{2}, \mathrm{a}_{7}, \mathrm{a}_{8}\right\}$ and $\mathrm{P}_{5}=\left\{\mathrm{a}_{3}, \mathrm{a}_{6}\right\}$ & 0.904758 \\
\hline
\end{tabular}

Table 11: The best disjoint paths for each Cs value using algorithm II To Example A

\begin{tabular}{lll}
\hline Cs & The best disjoint pair & $\mathrm{R}_{\mathrm{d}, \mathrm{T}, \mathrm{Cs}}$ \\
\hline 1 & $\mathrm{P}_{2}=\left\{\mathrm{a}_{1}, \mathrm{a}_{5}, \mathrm{a}_{8}\right\}$ and $\mathrm{P}_{5}=\left\{\mathrm{a}_{3}, \mathrm{a}_{6}\right\}$ & 0.948313 \\
2 & $\mathrm{P}_{2}=\left\{\mathrm{a}_{1}, \mathrm{a}_{5}, \mathrm{a}_{8}\right\}$ and $\mathrm{P}_{5}=\left\{\mathrm{a}_{3}, \mathrm{a}_{6}\right\}$ & 0.948313 \\
3 & $\mathrm{P}_{4}=\left\{\mathrm{a}_{1}, \mathrm{a}_{2}, \mathrm{a}_{7}, \mathrm{a}_{8}\right\}$ and $\mathrm{P}_{5}=\left\{\mathrm{a}_{3}, \mathrm{a}_{6}\right\}$ & 0.904758 \\
\hline
\end{tabular}

Table 12: The best disjoint paths for each Cs value using algorithm II to example B.

\begin{tabular}{llr}
\hline Cs & The best disjoint pair & $\mathrm{R}_{\mathrm{d}, \mathrm{T}, \mathrm{Cs}}$ \\
\hline 10 & $\mathrm{P}_{1}=\left\{\mathrm{a}_{1}, \mathrm{a}_{2}, \mathrm{a}_{3}\right\}$ and $\mathrm{P}_{6}=\left\{\mathrm{a}_{11}, \mathrm{a}_{12}, \mathrm{a}_{13}\right\}$ & 0.841395 \\
20 & $\mathrm{P}_{1}=\left\{\mathrm{a}_{1}, \mathrm{a}_{2}, \mathrm{a}_{3}\right\}$ and $\mathrm{P}_{6}=\left\{\mathrm{a}_{11}, \mathrm{a}_{12}, \mathrm{a}_{13}\right\}$ & 0.841395 \\
40 & $\mathrm{P}_{1}=\left\{\mathrm{a}_{1}, \mathrm{a}_{2}, \mathrm{a}_{3}\right\}$ and $\mathrm{P}_{6}=\left\{\mathrm{a}_{11}, \mathrm{a}_{12}, \mathrm{a}_{13}\right\}$ & 0.841395 \\
50 & $\mathrm{P}_{6}=\left\{\mathrm{a}_{11}, \mathrm{a}_{12}, \mathrm{a}_{13}\right\}$ and $\mathrm{P}_{8}=\left\{\mathrm{a}_{15}, \mathrm{a}_{16}, \mathrm{a}_{17}\right\}$ & 0.834777 \\
\hline
\end{tabular}

Table 13: Comparison results

\begin{tabular}{|c|c|c|c|c|c|}
\hline \multicolumn{3}{|c|}{ Example A } & \multicolumn{3}{|c|}{ Example B } \\
\hline Cs & $\begin{array}{l}\text { Reliability using } \\
\text { Algorithm I }\end{array}$ & $\begin{array}{l}\text { Reliability using } \\
\text { Algorithm II }\end{array}$ & Cs & $\begin{array}{l}\text { Reliability using } \\
\text { Algorithm I }\end{array}$ & $\begin{array}{l}\text { Reliability using } \\
\text { Algorithm II }\end{array}$ \\
\hline 1 & 0.771638 & 0.841395 & 10 & 0.91 & 0.841395 \\
\hline 2 & 0.93312 & 0.841395 & 20 & 0.91 & 0.841395 \\
\hline \multirow[t]{2}{*}{3} & 0.77824 & 0.841395 & 40 & 0.822451 & 0.841395 \\
\hline & & & 50 & 0.789727 & 0.834777 \\
\hline
\end{tabular}

\section{Conclusion}

Taking into account the required system capacity $(C s)$ and the transmission time $(T)$, the paper presented an algorithm to determine the set of disjoint paths that would simultaneously carry the data and then, generate all $(d, T, C s)$-DMPs to calculate the system reliability $R_{(d, T, C s)}$. Moreover, in this study, the benefits of using the system capacity to determine the available paths that can be used to send data have been investigated. In addition, the computational complexity taken by the algorithm to generate all solutions is $O(\delta n)$.

\section{Acknowledgment}

We would like to thank all the parties involved in this research work.

\section{Ethics}

The author confirm that they have thoroughly seen the content of the paper and do not find any conflict of interest and ethical issues.

\section{References}

Aggarwal, K.K., Y.C. Chopra and J.S. Bajwa, 1982. Capacity consideration in reliability analysis of communication system. IEEE Trans. Reliability, 31: 177-181. DOI: 10.1109/TR.1982.5221290

Alexopoulos, C., 1995. A Note on state-space decomposition methods for analyzing stochastic flow networks. IEEE Trans. Reliability, 44: 354-357. DOI: $10.1109 / 24.387394$

Chen, G.H. and Y.C. Hung, 1993. On the quickest path problem. Inform. Process. Lett., 46: 125-128. DOI: 10.1016/0020-0190(93)90057-G 
Chen, G.H. and Y.C. Hung, 1994. Algorithms for the constrained quickest path problem and the enumeration of quickest paths. Comput. Operat. Res., 21: 113-118. DOI: 10.1016/0305-0548(94)90045-0

Chin, Y.H. and Y.L. Chen, 1990. The quickest path problem. Comput. Operat. Res., 17: 153-161. DOI: 10.1016/0305-0548(90)90039-A

El Khadiri, M. and W.C. Yeh, 2016. An efficient alternative to the exact evaluation of the quickest path flow network reliability problem. Comput. Operat. Res., 76: 22-32. DOI: $10.1016 /$ j.cor.2016.06.010

Hassan, M.R., 2012. Reliability evaluation of stochasticflow network under quickest path and system capacity constraints. Int. J. Comput. Netw., 4: 98-103.

Janan, X., 1985. On multistate system analysis. IEEE Trans. Reliability, r-34: 329-337.

Jane, C.C. and Y.W. Laih, 2017. Distribution and reliability evaluation of max-flow in dynamic multi-state flow networks. Eur. J. Operat. Res., 259: 1045-1053. DOI: 10.1016/j.ejor.2016.12.030

Lee, D.T. and E. Papadopoulou, 1993. The all-pairs quickest path problem. Inform. Process. Lett., 45: 261-367. DOI: 10.1016/0020-0190(93)90214-T

Lin, Y.K., 2003. Extend the quickest path problem to the system reliability evaluation for a stochastic-flow network. Comput. Operat. Res., 30: 567-575.

DOI: 10.1016/S0305-0548(02)00025-4

Lin, Y.K., 2009a. Time version of the shortest path problem in a stochastic-flow network. J. Comput. Applied Math., 228: 150-157. DOI: 10.1016/j.cam.2008.09.003

Lin, Y.K., 2009b. Routing policy of stochastic-flow networks under time threshold and budget constraint. Expert Syst. Applic., 36: 6076-6081. DOI: 10.1016/j.eswa.2008.07.080

Lin, Y.K., 2010. Reliability of $k$ separate minimal paths under both time and budget constraints. IEEE Trans. Reliability, 59: 183-190.

DOI: 10.1109/TR.2009.2035794

Lin, Y.K., 2011a. Network reliability of a time-based multistate network under spare routing with $\rho$ minimal paths. IEEE Trans. Reliability, 60: 61-69. DOI: 10.1109/TR.2010.2103594

Lin, Y.K., 2011b. Stochastic flow networks via multiple paths under time threshold and budget constraint. Comput. Math. Applic., 62: 2629-2638.

DOI: $10.1016 /$ j.camwa.2011.08.002

Locks, M.O., 1980. Recursive disjoint products, inclusion-exclusion and min-cut approximations. IEEE Trans. Reliability, R-29: 425-436.

DOI: $10.1109 /$ TR. 1980.5220885
Martins, E.D.Q.V. and J.L.E.D. Santos, 1997. An algorithm for the quickest path problem. Operat. Res. Lett., 20: 195-198. DOI: 10.1016/S0167-6377(97)00008-4

Sedeno-Noda, A. and J.D. Gonzalez-Barrera, 2014. Fast and fine quickest path algorithm. Eur. J. Operat. Res., 238: 596-606. DOI: $10.1016 /$ j.ejor.2014.04.028

Yeh, W.C., 2015. A fast algorithm for quickest path reliability evaluations in multi-state flow networks. IEEE Trans. Reliability, 64: 1175-1184. DOI: 10.1109/TR.2015.2452580

Yeh, W.C., L.E. Lin, Y.C. Chou and Y.C. Chen, 2013. Optimal routing for multi-commodity in multistate flow network with time constraints. Qual. Technol. Quant. Manage., 10: 161-177. DOI: $10.1080 / 16843703.2013 .11673314$

Zuo, M.J., Z. Tian and H.Z. Huang, 2007. An efficient method for reliability evaluation of multistate networks given all minimal path vectors. IIE Trans., 39: 811-817. DOI: 10.1080/07408170601013653

\section{Acronyms}

DMPs Disjoint minimal paths.

SFN Stochastic-flow network.

QPP Quickest path problem.

\section{Notations}

G(A, N, M) SFN with a set of nodes N, a set of $\operatorname{arcs} A=$ $\left\{a_{i} \mid 1 \leq i \leq n\right\}$ and $\mathrm{M}=\left\{M^{1}, M^{2}, \ldots, M^{n}\right\}$ with $M^{i}$ (an integer) being the maximum capacity of each $\operatorname{arc} a_{i}$.

$X \quad$ Capacity vector; $X=\left(x_{1}, x_{2}, \ldots, x_{n}\right)$.

MPs Minimal paths.

$\mathrm{MP}_{\mathrm{j}} \quad$ A minimal path no. $j ; j=1,2, \ldots, \omega$.

$\mathrm{TP}_{\mathrm{j}} \quad$ The transmission time of path $P_{j}$.

$\mathrm{CP}_{\mathrm{j}} \quad$ The maximum capacity of $P_{j}$.

d The given demand, units of data to be transmitted.

T The time limit, units of time.

Cs The required system capacity, given in units of cost.

$l_{i} \quad$ The lead time of arc $a_{i}$.

$R_{d, T, C s} \quad$ System reliability for the given demand $d$ under T and Cs.

\section{Nomenclature}
$X \leq Y \quad X=\left(x_{1}, x_{2}, \ldots, x_{n}\right) \leq Y=\left(y_{1}, y_{2}, \ldots, y_{n}\right)$, if $x_{i} \leq y_{i}$ for $i=1,2, \ldots, n$
$X<Y \quad X \leq Y$, when $x_{i}<y_{i}$ for at least one i

Lower Vector $X \in \Gamma$ is a lower vector if there is no $Y \in \Gamma$ such that $Y<X$. 
Moatamad Refaat Hassan / American Journal of Applied Sciences 2018, 15 (5): 251.260 DOI: 10.3844/ajassp.2018.251.260

\section{Assumptions}

1. The capacity of each arc $a_{i}$ is an integer-valued random variable, which takes values $0<1<2<\ldots M^{i}$ according to a given distribution.
2. Each node is perfectly reliable.

3. The capacities of different components are statistically independent. 